\title{
Managerial leadership for research use in nursing and allied health care professions: a systematic review
}

Wendy A. Gifford ${ }^{1 *} \mathbb{D}$, Janet E. Squires ${ }^{1,2}$, Douglas E. Angus ${ }^{3}$, Lisa A. Ashley ${ }^{4}$, Lucie Brosseaư ${ }^{5}$, Janet M. Craik ${ }^{6}$, Marie-Cécile Domeca ${ }^{7}$, Mary Egan ${ }^{5,8}$, Paul Holyoke ${ }^{9}$, Linda Juergensen ${ }^{1,10}$, Lars Wallin ${ }^{11,12,13}$, Liquaa Wazni ${ }^{1}$ and lan D. Graham ${ }^{12,14}$

\begin{abstract}
Background: Leadership by point-of-care and senior managers is increasingly recognized as critical to the acceptance and use of research evidence in practice. The purpose of this systematic review was to identify the leadership behaviours of managers that are associated with research use by clinical staff in nursing and allied health professionals.

Methods: A mixed methods systematic review was performed. Eight electronic bibliographic databases were searched. Studies examining the association between leadership behaviours and nurses and allied health professionals' use of research were eligible for inclusion. Studies were excluded if leadership could not be clearly attributed to someone in a management position. Two reviewers independently screened abstracts, reviewed full-text articles, extracted data and performed quality assessments. Narrative synthesis was conducted.

Results: The search yielded 7019 unique titles and abstracts after duplicates were removed. Three hundred five full-text articles were reviewed, and 31 studies reported in 34 articles were included. Methods used were qualitative $(n=19)$, cross-sectional survey $(n=9)$, and mixed methods $(n=3)$. All studies included nurses, and six also included allied health professionals. Twelve leadership behaviours were extracted from the data for point-of-care managers and ten for senior managers. Findings indicated that managers performed a diverse range of leadership behaviours that encompassed change-oriented, relation-oriented and task-oriented behaviours. The most commonly described behavior was support for the change, which involved demonstrating conceptual and operational commitment to research-based practices.

Conclusions: This systematic review adds to the growing body of evidence that indicates that manager-staff dyads are influential in translating research evidence into action. Findings also reveal that leadership for research use involves change and task-oriented behaviours that influence the environmental milieu and the organisational infrastructure that supports clinical care. While findings explain how managers enact leadership for research use, we now require robust methodological studies to determine which behaviours are effective in enabling research use with nurses and allied health professionals for high-quality evidence-based care.
\end{abstract}

Trial registration: PROSPERO CRD42014007660

Keywords: Leadership, Managers, Administrators, Research use, Evidence-based practice, Allied health, Nursing

\footnotetext{
* Correspondence: wgifford@uottawa.ca

${ }^{1}$ Faculty of Health Sciences, School of Nursing, University of Ottawa, Ottawa,

Ontario, Canada

Full list of author information is available at the end of the article
}

(C) The Author(s). 2018 Open Access This article is distributed under the terms of the Creative Commons Attribution 4.0 International License (http://creativecommons.org/licenses/by/4.0/), which permits unrestricted use, distribution, and reproduction in any medium, provided you give appropriate credit to the original author(s) and the source, provide a link to the Creative Commons license, and indicate if changes were made. The Creative Commons Public Domain Dedication waiver (http://creativecommons.org/publicdomain/zero/1.0/) applies to the data made available in this article, unless otherwise stated. 


\section{Background}

The use of research evidence in clinical practice has advanced healthcare delivery from unpredictable and unproven practices to treatments based on rigorous research evidence to improve outcomes [1, 2]. However, research use continues to be a challenge across all healthcare disciplines and settings [3-5], with over two-thirds of implementation efforts deemed unsuccessful [6]. For example, a recent cross-sectional survey revealed that only $12 \%$ of nurses and allied health professionals in the European Society of Cardiology used research-based evidence from guidelines in their practice [7]. While much of the implementation research focusses on individual practitioners [8], leadership within the organisational context is increasingly recognized as a strong influencing factor on the acceptance and use of research evidence in practice [9]. In the present study, the concept of using research in clinical practice is based on Sackett et al.'s (1997) widely accepted definition of evidence-based medicine: 'the conscientious, explicit and judicious use of current best evidence in making decisions about the care of individual clients' [10].

With the growing recognition of the importance of leadership in implementation efforts, the mechanisms by which leadership influences research use are receiving increasing attention $[9,11]$. Leadership has been defined and studied in many ways across disciplines. In this study, we use a highly used definition of leadership as a process that influences, motivates, and enables others [12]. Behavioural leadership theory suggests that effective leadership involves behaviours from three broad conceptual categories: (1) change-oriented, (2) relation-oriented and (3) task-oriented behaviours [13-15]. Change-oriented behaviours are concerned primarily with providing vision and direction for innovation, creating a sense of need, and building coalitions to support change. Relation-oriented behaviours involve supporting, developing and recognizing others with the primary objective to increase the quality of human resources and relations, thereby increasing trust, cooperation and commitment amongst members. Task-oriented behaviours include clarifying roles, planning, monitoring performance and outcomes and using resources efficiently [13-15].

Transformational and transactional leadership theories are well known and widely researched leadership approaches $[16,17]$. Transformational leadership is the degree to which a leader inspires and motivates others to follow an ideal or a particular course of action [16], while transactional leadership involves the provision of incentives, rewards and monitoring to meet quality standards [17]. Dimensions of both transformational and transactional leadership align with the leadership behaviours in task-oriented, relation-oriented and change-oriented conceptual categories. For example, transformational leadership can influence attitudes towards research use through relations and change-oriented behaviours of envisioning change, facilitating collective learning and supporting and recognizing efforts, whereas transactional leadership aligns with task-oriented behaviours of clarifying roles, planning and monitoring operations to accomplish work in an efficient and reliable way. Consistent with behavioural leadership theory, the relevance of each behavior depends on the aspect of the situation and the context of the implementation efforts [13].

The leadership behaviours of point-of-care and senior managers have been shown to strongly influence nurses and allied healthcare professionals' use of research evidence, while lack of leadership is consistently identified as a major barrier to implementation [18-21]. Managers are employees who oversee staff, have budgetary accountabilities [22] and play a role in ensuring high-quality patient care [23, 24]. Point-of-care managers (e.g. head nurses, managers or supervisors) are responsible for unit operations, with front-line staff reporting to them, while senior managers (e.g. administrators, directors, operating officers) have broader organisational responsibilities, with one or more managers typically reporting to them [25]. A recent American mixed-methods study examining implementation of an evidence-based innovation in social welfare organisations to reduce child maltreatment found that successful implementation was 17 times higher with strong leadership, and failure was associated with passive/ avoidant leadership [9].

Nurses and allied healthcare professionals constitute the largest proportion of the healthcare team and play a central role in ensuring high-quality and effective care delivery. Nurses are self-regulated professionals that deliver autonomous and collaborative care in health promotion, illness prevention and caring for ill, disabled and dying people [26]; they include registered nurses (RNs), licensed practical nurses (LPNs), registered practical nurses (RPNs), nurse practitioners and registered psychiatric nurses [27]. Allied health professionals are licenced to provide specific types of healthcare services but are not physicians or nurses [28]. While disciplines under the umbrella term 'allied health' vary [29], for purposes of this review they include physiotherapists (PTs), occupational therapists (OTs), speech-language pathologists (SLPs) and dietitians as defined a priori in the study protocol [30].

Managers are strategically positioned to support and facilitate nurses and allied health professionals' use of research evidence through organisational policies, procedures, systems and climates [9, 31]. A 2007 integrative literature review identified that managers used facilitative and regulatory behaviours to influence nurses to use research evidence, including support, policy revisions and clinical practice audits [32]. However, relevant literature has not been systematically synthesized for allied health professionals or updated for nurses, and little is 
known about healthcare managers' approaches to support their research use. Understanding leadership behaviours that advance research use is fundamental for designing interventions for organisations to improve healthcare delivery and patient outcomes.

The purpose of this systematic review was to synthesize evidence on the association between leadership behaviours of point-of-care and senior managers and research use by nurses and allied health professionals. The specific objectives were (1) to identify managers' leadership behaviours that are associated with research use by nurses and allied health professionals in clinical practice and, if studies permit, (2) to determine the effectiveness of interventions to develop leadership for facilitating research use by nurses and allied health professionals.

\section{Methods}

We conducted a mixed-methods systematic review to synthesize diverse forms of evidence related to point-ofcare and senior managers' leadership behaviors that are associated with nurses and allied health professionals' research use in clinical practice [30]. We used a systematic approach to synthesize quantitative, qualitative and mixed-methods results using methodological guidelines set forth by Grimshaw [33].

\section{Concepts and definitions}

Several forms of research use have been discussed in the literature, including instrumental, conceptual and symbolic $[34,35]$. We focussed on instrumental research use or the concrete application of research knowledge as we were interested in improved healthcare delivery through behaviour change in clinical practice. The evidence included guidelines, protocols, policies or procedures based explicitly on research. We defined leadership 'behaviours' as managerial activities and engagement practices that influence nurses and/or allied health providers to use research evidence in their clinical practice.

\section{Search strategy}

In collaboration with a health sciences librarian, we developed and implemented the search strategy, using eight electronic bibliographic databases (ABI Inform Global, CINAHL, Cochrane Database of Systematic Reviews, Cochrane Central Register of Controlled Trials, EMBASE, MEDLINE, Pedro, Proquest Nursing and Allied Health, PsycINFO) and covering all available published works up to June 2018. Keywords, and their synonyms and medical subject headings were used for leadership, management and research use in each database (see Additional file 1 for search strategy). Reference lists of included studies were assessed for relevant citations.

\section{Study inclusion/exclusion criteria}

Studies investigating managerial leadership behaviours and staff research use were included. To be included, studies needed to report on actual (not planned) instrumental research use and managerial leadership behaviours. Instrumental research use was expressed at the individual practitioner or unit levels and included settings classified as having high and low levels of research use. Evidence-based practice [36] was included if instrumental research use was studied separately from the multi-step process of constructing a clinical question and critically appraising the literature. Studies were excluded if leadership could not be clearly attributed to someone in a management position such as those using the terms 'leader,' 'senior nurse', 'hospital leadership' or 'organisational leadership' without identifying a management role, or if greater than $50 \%$ of the sample was not nurses or allied health professionals. Studies were limited to those published in English or French, the official languages of our research team, with no restrictions on country of origin or publication date.

\section{Types of studies}

Experimental (e.g. randomized controlled trials), quasiexperimental (e.g. pre/post-test), non-experimental (e.g. cross-sectional surveys), mixed-methods and qualitative designs were included. Commentaries, editorials and theses were excluded.

Quantitative studies had to propose a relationship between managerial leadership behaviours and staff research use and test it statistically, with instrumental research use as the dependent variable and leadership as the independent variable. Interventions must have involved front line or senior level managers for the purpose of influencing clinical staff use research in practice.

\section{Participants}

Nurses included RNs, LPNs, RPNs, nurse practitioners and registered psychiatric nurses; allied health professionals included PTs, OTs, SLPs and dieticians.

\section{Selection of studies}

Two reviewers independently screened all titles and abstracts identified in the database search for eligibility. Full-text copies were retrieved for all citations identified as potentially relevant or having insufficient information to make a decision. Retrieved articles were assessed for alignment with inclusion criteria independently by two team members; discrepancies were resolved through discussion and adjudication with senior research team members (WG, JES, IDG). 


\section{Quality assessment}

We used three tools to assess the methodological quality of included studies according to study design: (1) Quality Assessment and Validity Tool for Cross-sectional Studies, (2) Quality Assessment and Validity Tool for before/after Design studies and (3) Critical Appraisal Skills Programme (CASP) Qualitative Research Checklist. Two reviewers independently conducted the quality assessment on all included articles; disagreements were resolved through discussion with a third senior reviewer. We adopted a scoring system used in a previously published systematic review [37]: for each article, a rating score was derived by taking the number obtained in the quality rating and dividing it by the total number of possible points allowed, giving each paper a total quality rating between 0 and 1 . Articles were then classified as weak $(<0.50)$, moderate-weak (0.51-0.65), moderate-strong (0.66-0.79) or strong $(0.80-1.00)$. Mixed-methods studies were assessed with two corresponding tools.

Qualitative studies were assessed using the Critical Appraisal Skills Programme (CASP) Qualitative Research Checklist [38], which assesses methodology through ten questions on research aims, appropriateness of methodology, research design, recruitment strategy, data collection, adequate consideration of the relationship between researchers and participants, ethical issues, data analysis, clarity of findings and research value. Cross-sectional quantitative studies were assessed with the Quality Assessment and Validity Tool for Cross-sectional Studies [39], which focuses on reporting quality and methodological rigor in four domains: sample, measurement, statistical analysis and conclusion. Intervention studies were assessed using the Quality Assessment and Validity Tool for before/after Design studies, adapted from Cochrane Collaboration guidelines and used in other systematic reviews [40]. It focuses on six domains: sampling, design, control of confounders, data collection and outcome measurement, statistical analysis and conclusions as well as dropouts. No studies were excluded based on the quality assessment.

\section{Data extraction}

One reviewer extracted data from all included articles, a second reviewer verified for accuracy and a senior reviewer resolved discrepancies. Data were extracted on publication year, country, research purpose and objectives, research design, setting, data collection methods, sample size and participant characteristics, behaviours of managers (independent variables/concepts), managers' titles and characteristics, research use variables or concepts, analysis, and key findings regarding the relationship between managers' leadership behaviours and research use by nurses and allied health professionals.

\section{Data synthesis}

A narrative data synthesis was conducted using Popay et al.'s [41] procedures to produce a summary of the research studies. Qualitative study data on managers' behaviours were pooled and entered into NVivo qualitative software then inductively coded into descriptive themes using the primary authors' conceptualizations of the behaviours described. For example, if an author reported 'encourage' as a managerial behaviour, it was classified as 'encourage' in our analysis and not reclassified based on interpretations. We used a consensus technique to determine the descriptive themes and made inquiries to primary authors of included studies to clarify interpretations when needed.

Data extracted from quantitative studies were synthesized descriptively, identifying the dependent (research use) and the independent (leadership) variables. This was supplemented by extracting the direction and magnitude of effect for factors displaying statistical significance $(p<0.05)$ where provided. Additionally, when bivariate and multivariate statistics were both reported, the more robust multivariate findings were used.

Quantitative data were synthesized into descriptive themes using convergence when data from the two methods corroborated and expansion when additional insights were provided. The use of different leadership measures in the small number of quantitative studies prevented quantitative data from being combined for sub-group analysis or statistical assessment of the association between managers' leadership and research use.

Based on behavioral leadership theory, descriptive themes were deductively grouped into the three conceptual domains of leadership: change-oriented, relationoriented and task-oriented leadership behaviours [12-14]. Data categorization was initially completed by the research assistants and first author (WG); further synthesis and re-classifications occurred iteratively in group meetings with investigators (WG, IDG, JES, LW), where study data were compared and contrasted with descriptions of the behavioral leadership categories [14]. Findings were discussed with the entire research team until consensus was reached.

We did not analyze studies for the effectiveness of leadership interventions on research use by nurses and allied health professionals (objective two) because of the lack of experimental studies found in the review. The limited number of studies found also prevented us from conducting sub-group analysis for professional group, sector, or types of instrumental research use (i.e. research use or guideline use). While insufficient evidence was found to reach definitive conclusions regarding leadership behaviours associated with research use, findings from all included studies were narratively synthesized to provide a summary of the types of behaviours studied. 


\section{Results}

The database search yielded 7019 unique titles and/or abstracts after duplicate removal, with 305 identified as potentially relevant and retrieved in full text. Of those, 271 did not meet our inclusion criteria: 158 lacked instrumental use of research evidence in clinical practice by nurses or allied health professionals (for example studies about barriers and facilitator pre-implementation), 60 did not have behaviours performed by managers, 37 quantitative studies had no statistical evaluation of instrumental research use and a leadership measure and 17 had a sample with $<50 \%$ nurses or allied health professionals. Thirty-one studies represented in 34 articles met our inclusion criteria and were included in this review (Fig. 1).

\section{Description of studies}

Of the 31 studies, 9 reported cross-sectional surveys [4250], 3 had mixed-methods designs [31, 51, 52], and 19 had qualitative designs $[5,53-73]$. The mixed-methods studies included qualitative data with either a survey, experimental pilot, or quasi-experimental trial. Studies were conducted in Canada $(n=14)$ [31, 46, 48, 53-60, 66, 68, 70], Sweden $(n=6)$ [43-45, 47, 62, 73], USA $(n=5)$ [5, 42, 51, 52, 69], China $(n=1)$ [63, 64], Mongolia $(n=1)$ [65], Netherlands $(n=1)$ [71] and one study in multiple European countries [61]. Studies had different healthcare settings with 18 conducted in acute care hospitals [5, 27, 42, 46-49, 52-54, 56-59, 62-64, 67, 69, 72, 73], three in nursing homes [50-52], three in the community [31, 43, 44], one in each family health centre [65] and rehabilitation centre [70] and five had a combination $[45,55,60,68,71]$. All studies included nurses as participants with 20 exclusively targeting nurses (65\%), six also included allied health practitioners $[43,51,57$, $60,67]$ and eight also included other health providers such as physicians $[51,58,59,63-65,68,71,72]$ and
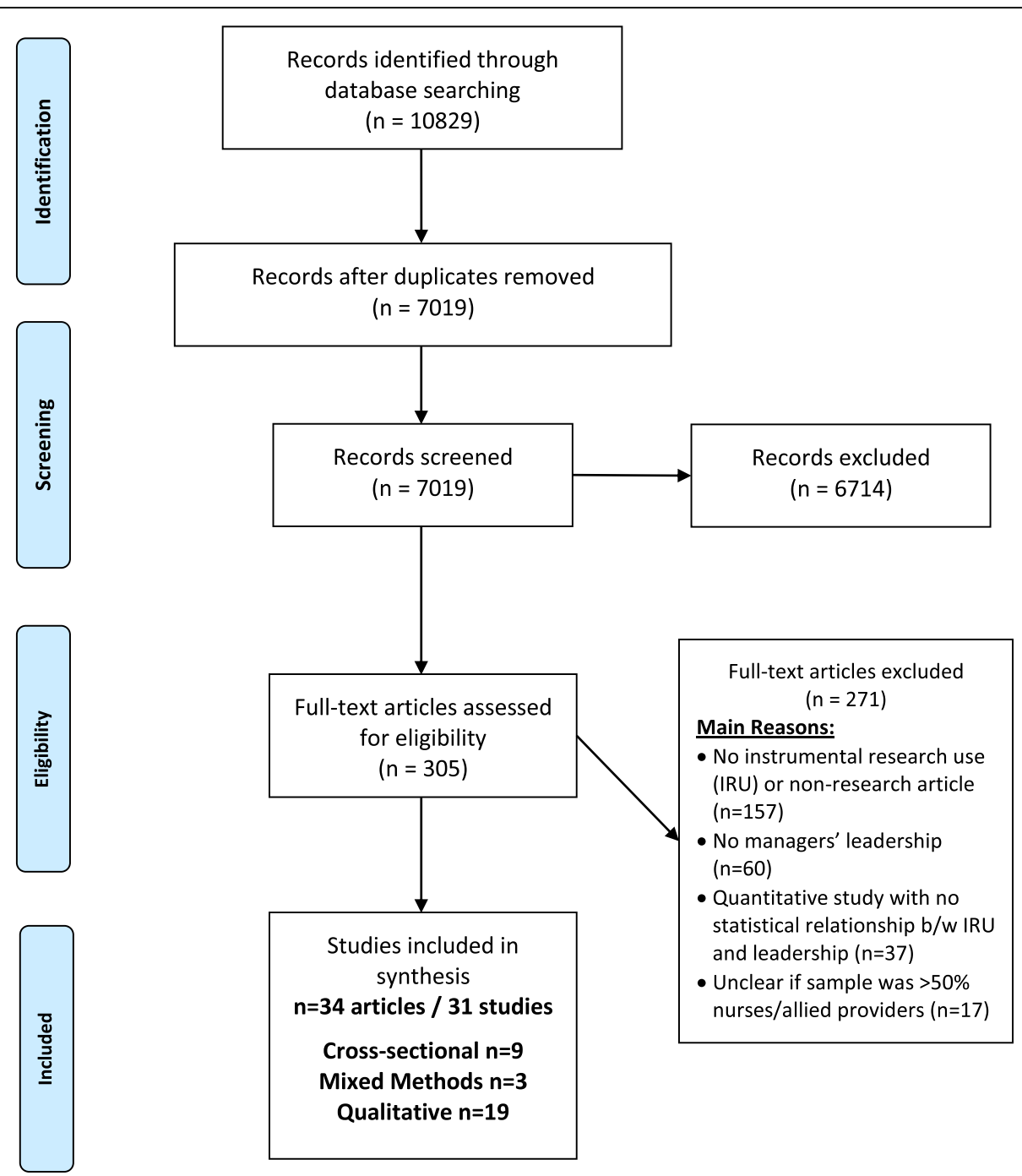

Fig. 1 PRISMA diagram of study selection 
healthcare aids [50, 68]. Characteristics of included studies are shown in Table 1.

The total number of participants in the combined studies was 5840 nursing staff (including nursing assistants or healthcare aids), 332 point-of-care managers, 190 physicians and or other healthcare providers, 129 senior leaders and 110 allied health professionals. Participants of the 11 studies that reported gender $[43-50,57,63,64,69]$ are as follows: $92 \%$ were female and $8 \%$ male.

Over half the studies $(n=21,68 \%)$ were published in the past 5 years (2013-2018) [5, 45, 47-52, 54, 57-59, 61, 6373 ] with the remainder published in the preceding 10 years (2003 and 2012) [31, 42-46, 55, 56, 60, 62]. The earliest study was published in 2003 [39], the number peaked in $2017(n=6)[49,63,67,68,70,73]$, and two were published in early 2018, when the search ended [50,64].

\section{Measures of research use}

The dependent variable of instrumental research use was measured through a single-item score on a 5 -point frequency scale, capturing how often participants use research-based practices when caring for patients $(1=$ less than $10 \%$; $5=$ almost $100 \%)[46,48,49]$, (scoring not stated [50]). A mean score of nine items [44] and a single item [43] from the Research Utilization Questionnaire measuring participants' agreement to using research findings in daily practice on a 5 -point Likert scale ( $1=$ strongly disagree to $5=$ strongly agree $)$.

Implementation of specific guideline recommendations was the dependent variable in five studies [31, 42, 47, 51, 52]. Forberg et al. [47] measured adherence to six guideline-based practices on a 5-point Likert scale $(1=$ never to $5=$ always $)$ and dichotomized each practice as always or not-always occurring, whereas Ball [42] measured how often participants perceived they followed guideline-based practice at four levels: always (100\%), often (50-99\%), sometimes $(<50 \%)$ and never. In the mixed-methods studies, survey scores [51], observations [52] and chart audits [31] determined the extent of guideline-based practices. Qualitative studies investigated implementation of specific guideline recommendations [54-62] or research-based practices [53].

\section{Measures of leadership \\ Point-of-care managers}

In ten studies, cross-sectional survey data was used to investigate statistical associations between research use and managers' leadership behaviors: six with point-of-care managers and two with senior managers. Different conceptual aspects of leadership were measured across eight of these studies. Leadership concepts were measured through the Alberta Context Tool $(n=3)$ [46-49], Research Utilization Questionnaire $(n=2)$ [43, 44], QPS Nordic scale $(n=1)$ [45], Managers' Support and
Coaching Conversation scales [50], and a survey specially developed for the study $(n=2)$ [42, 51]. Details of the measures used, statistical effects and direction and magnitude of the effect (if known) are presented in Table 1.

The Alberta Context Tool (ACT) was used to measure leadership in four of the included studies [46-49]. Leadership is measured as a mean score on a 5-point Likert scale of six items measuring the unit-level actions of formal leaders. The six leadership items reflect emotionally intelligent leadership and include: focussing on successes; looking for feedback; calmly handling stress; listening, acknowledging and responding; actively mentoring and coaching, and resolving conflicts [46-48].

The Research Utilization Questionnaire (RUQ) was used to measure leadership in two studies led by the same author [43, 44]. Leadership was measured using a single item on a 5-point Likert scale assessing leadership support for research utilisation. Another study by the same author used the QPS Nordic scale to measure three dimensions of leadership with six items [45]. Scores were dichotomized as high and low-quality leadership based on the dimensions: 1) social support, which involved a willingness to listen and help staff with task-related problems; 2) empowering leadership, which involved encouraging staff; and 3) fair leadership, which involved work-distribution and fair treatment of others.

Two mixed-methods studies involved leadership interventions directed at unit level managers, using qualitative data to provide insights into managers' leadership behaviors [31, 52]. The three-month intervention in Gifford et al. [31] included planning, developing an action plan and increasing communication with staff; whereas the intervention in Rangachari et al. [52] saw managers engage in weekly communications about central venous lines clinical audit results and processes for change over 52 weeks.

\section{Senior managers}

Two studies developed surveys that included measures of senior managers' leadership in implementation of research-based practices [42, 51]. Ball [42] measured leadership support using three items (support for clinical practice, policies and procedures) from a 79-item tool. Similarly, Balbale et al. [51] used three items related to managers' provision of adequate resources and training (number of survey items not revealed).

\section{Quality assessment}

Of the 31 studies reviewed, 20 were rated as strong (65\%) [5, 46, 48, 53-73], seven were high-moderate (23\%) [31, 42-45, 50, 52], two (6\%) were low-moderate $[47,49]$ and one (3\%) was weak [51]. All 19 qualitative articles [5, 53-73] and two of seven cross-sectional studies rated strong $[46,48]$. From the nine cross-sectional 


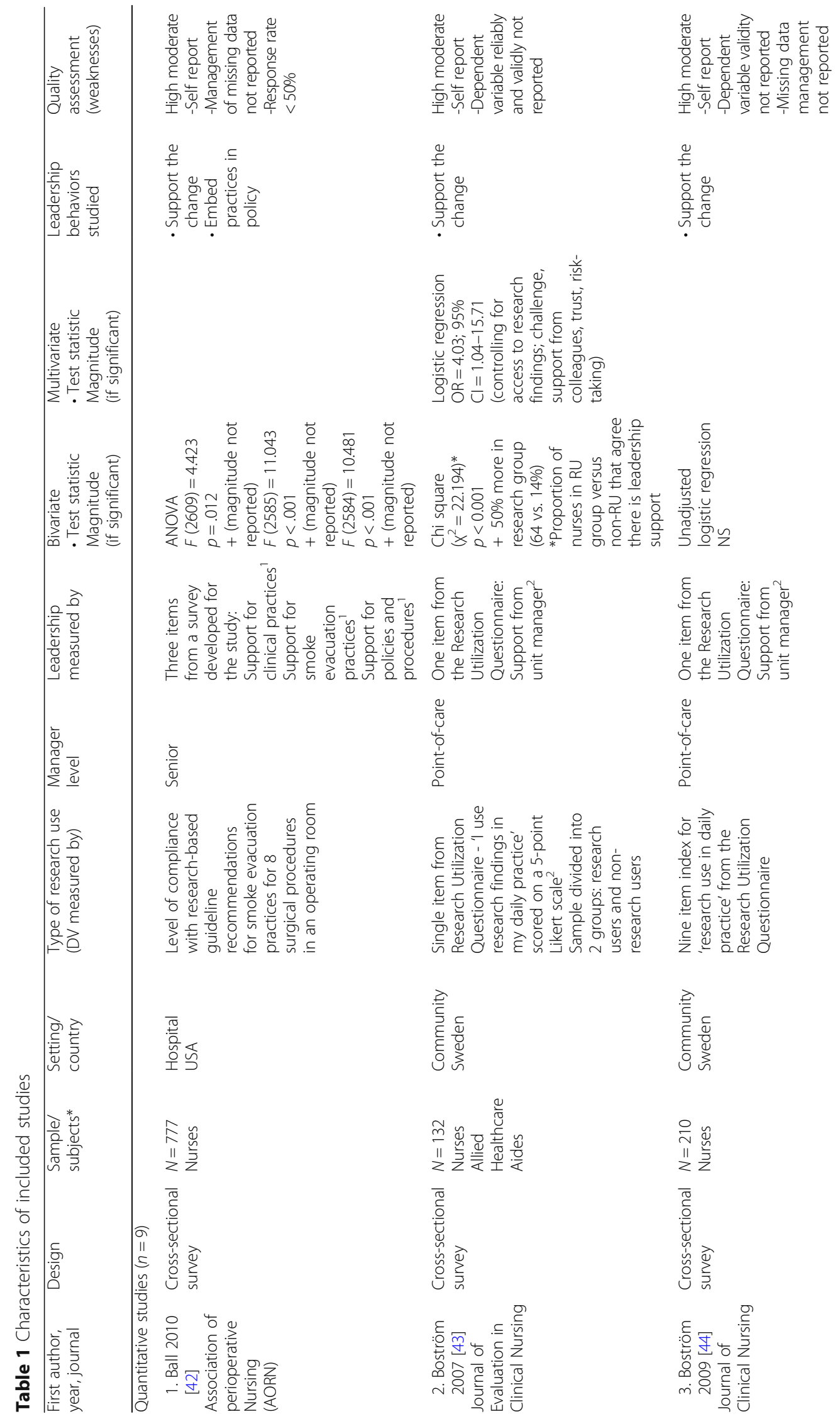




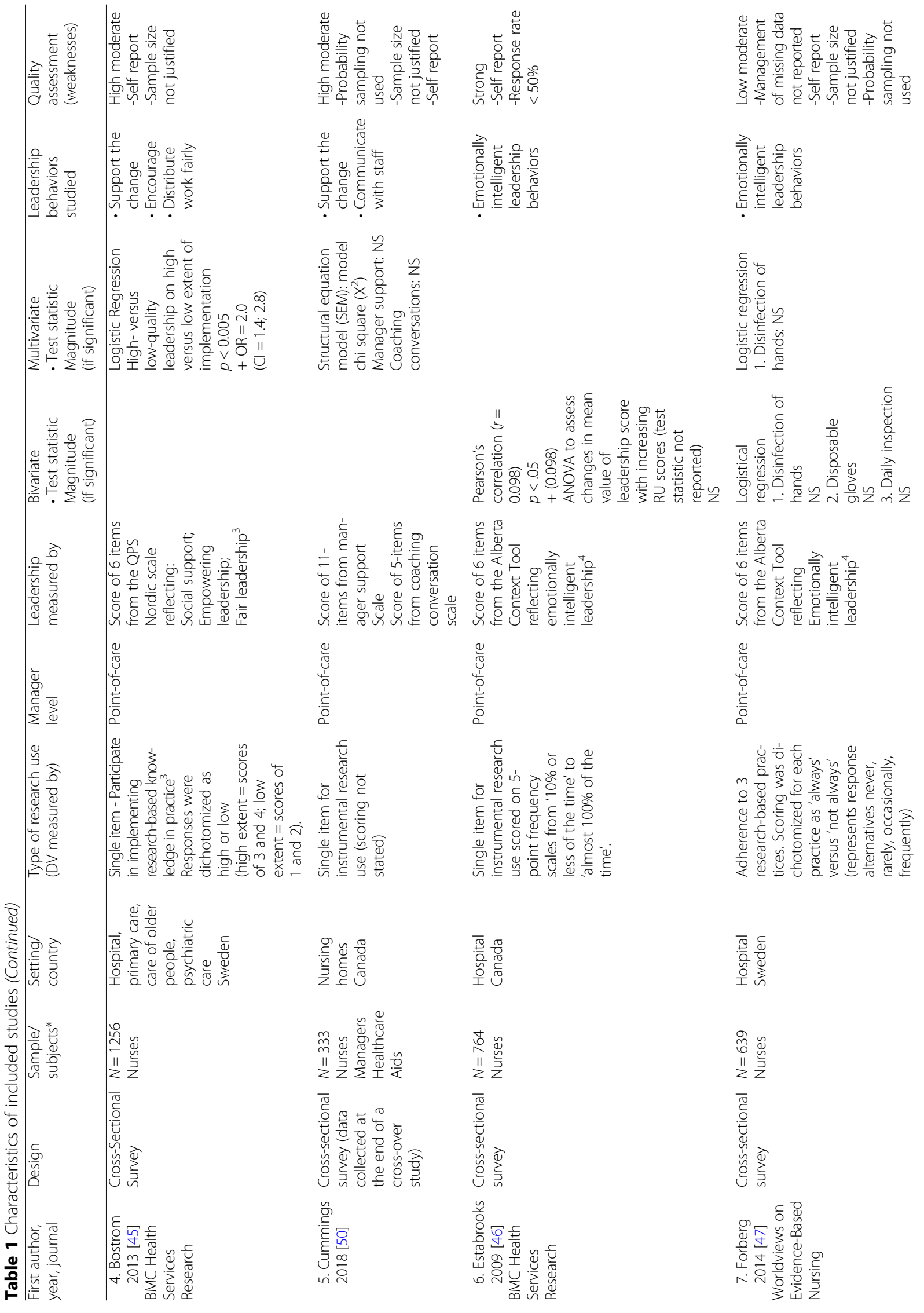




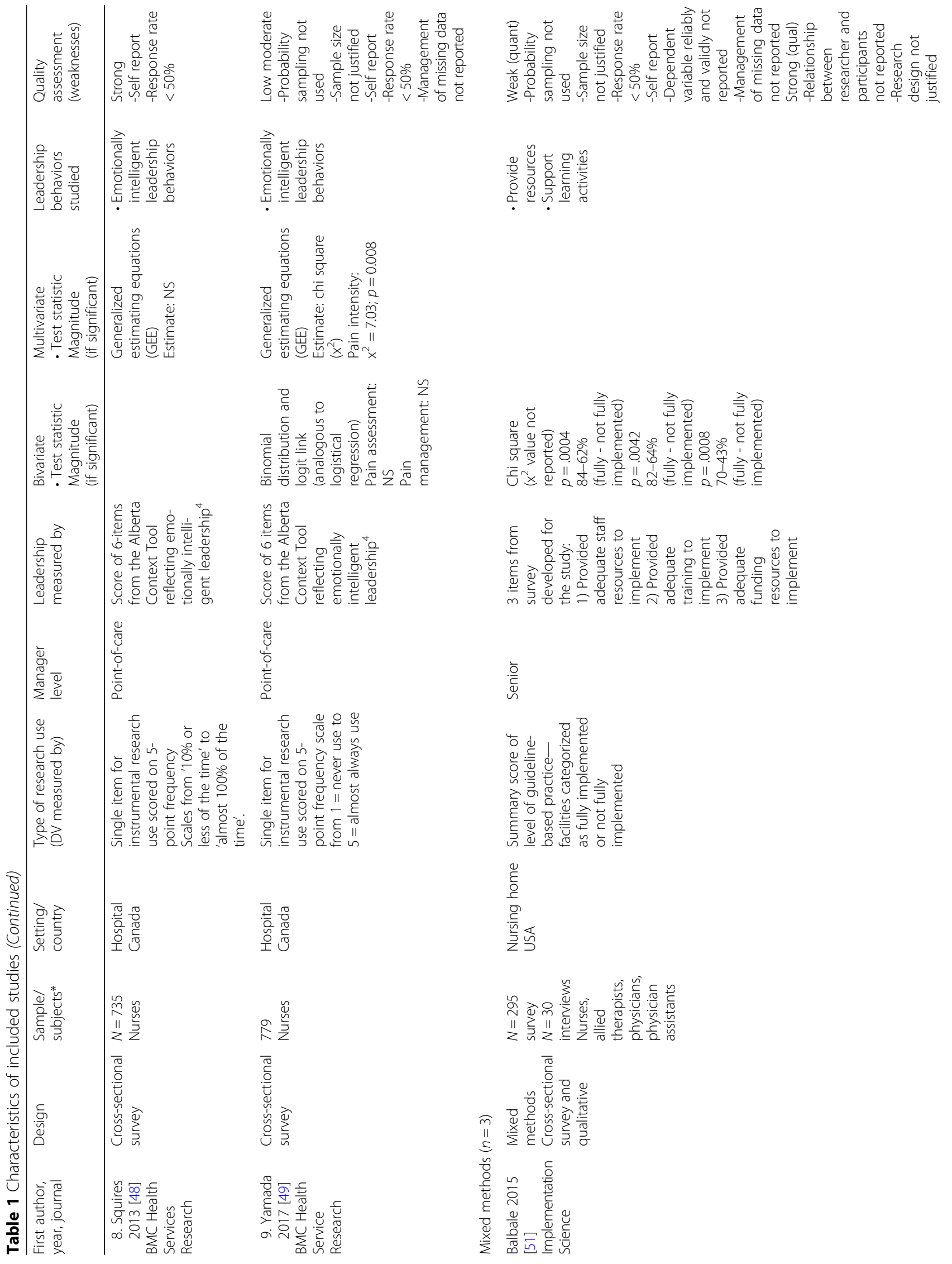




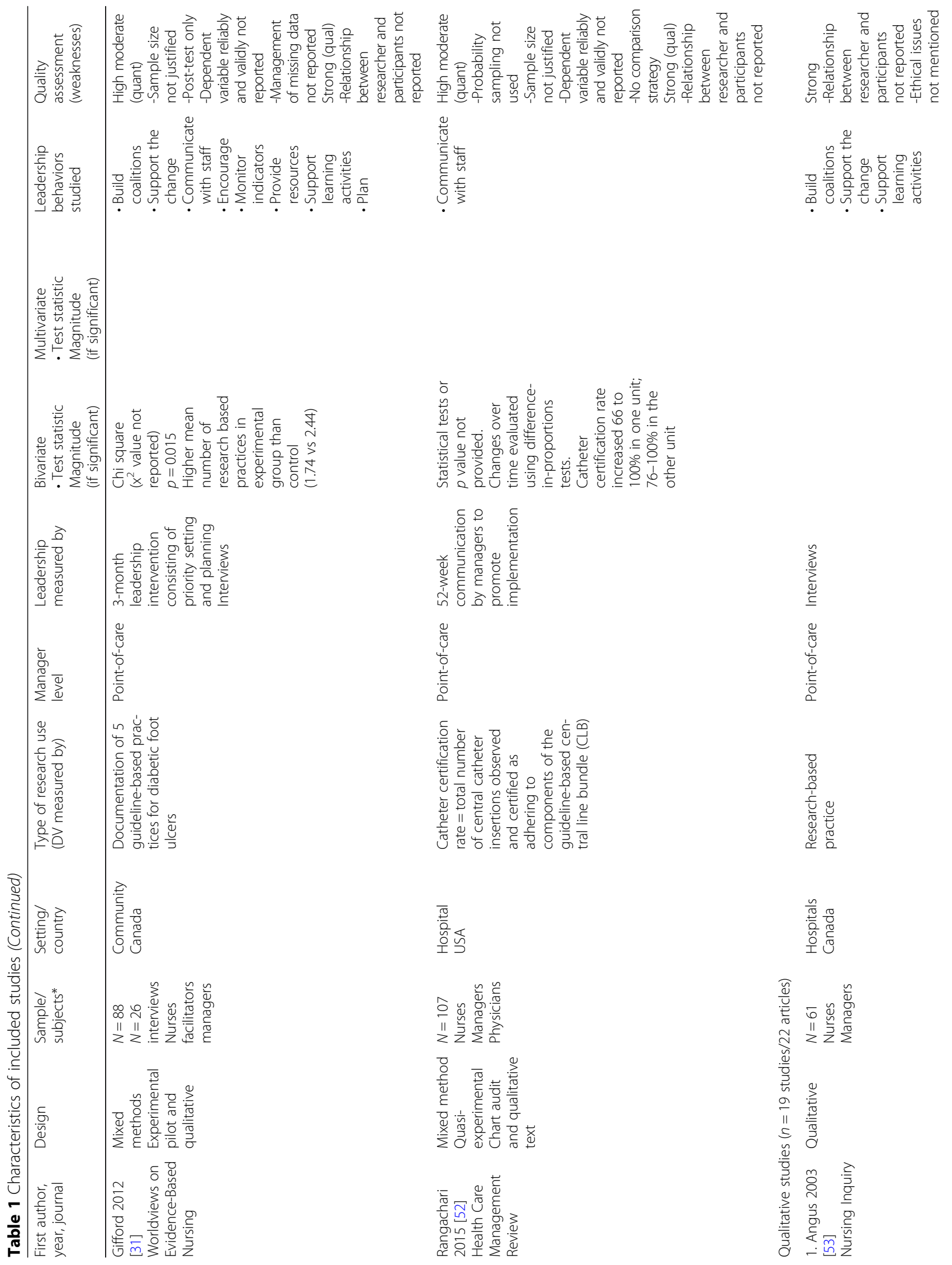




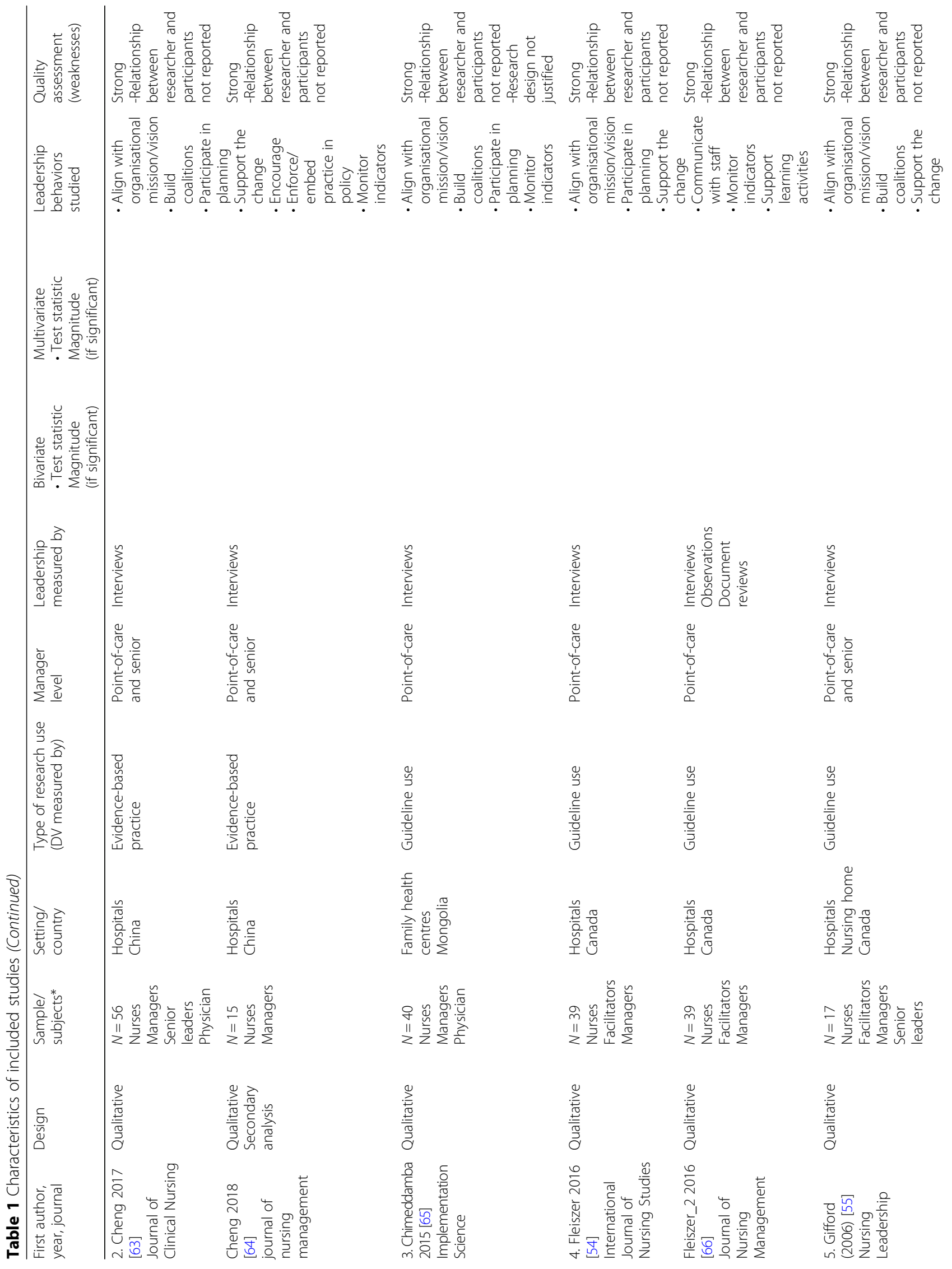




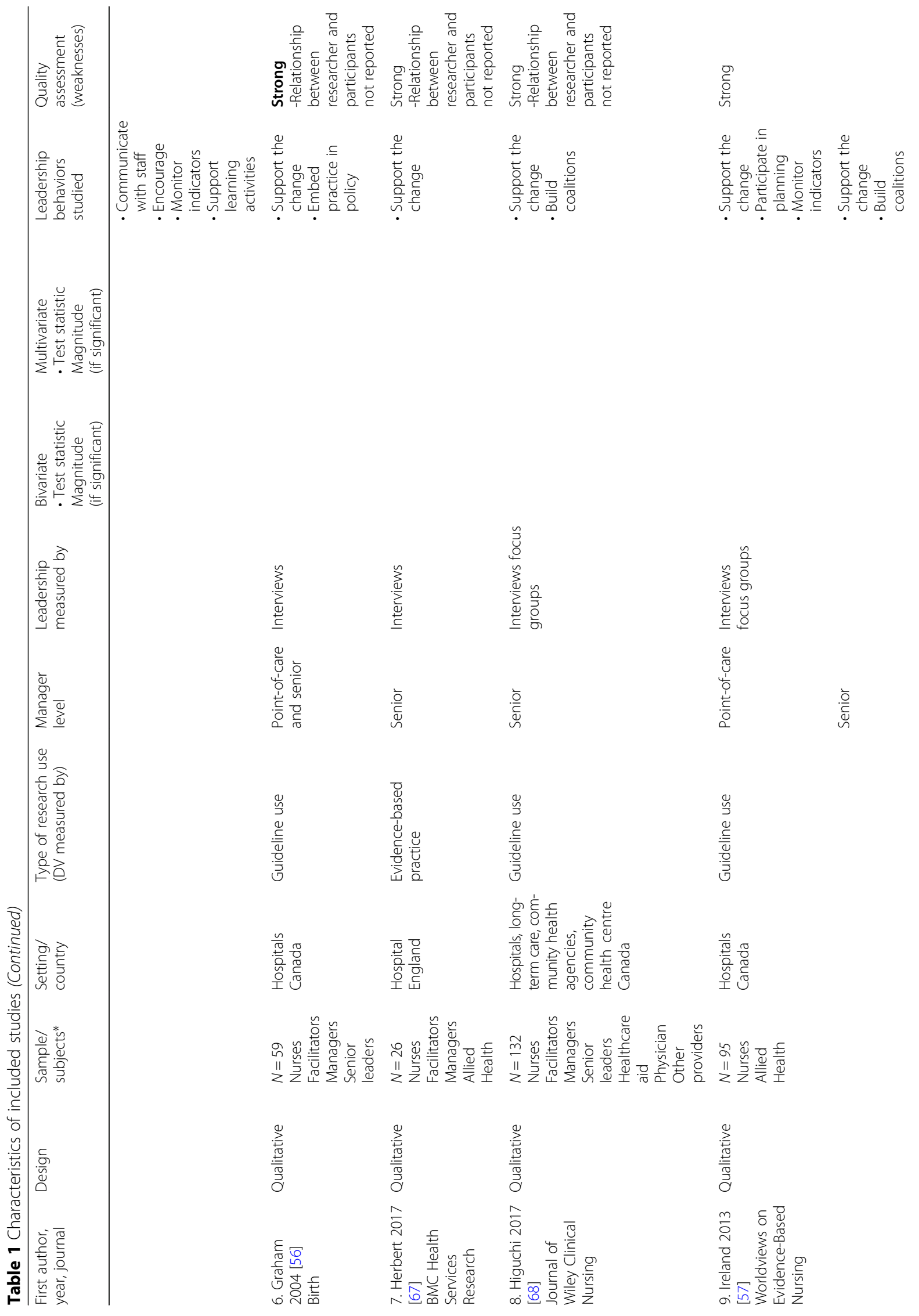




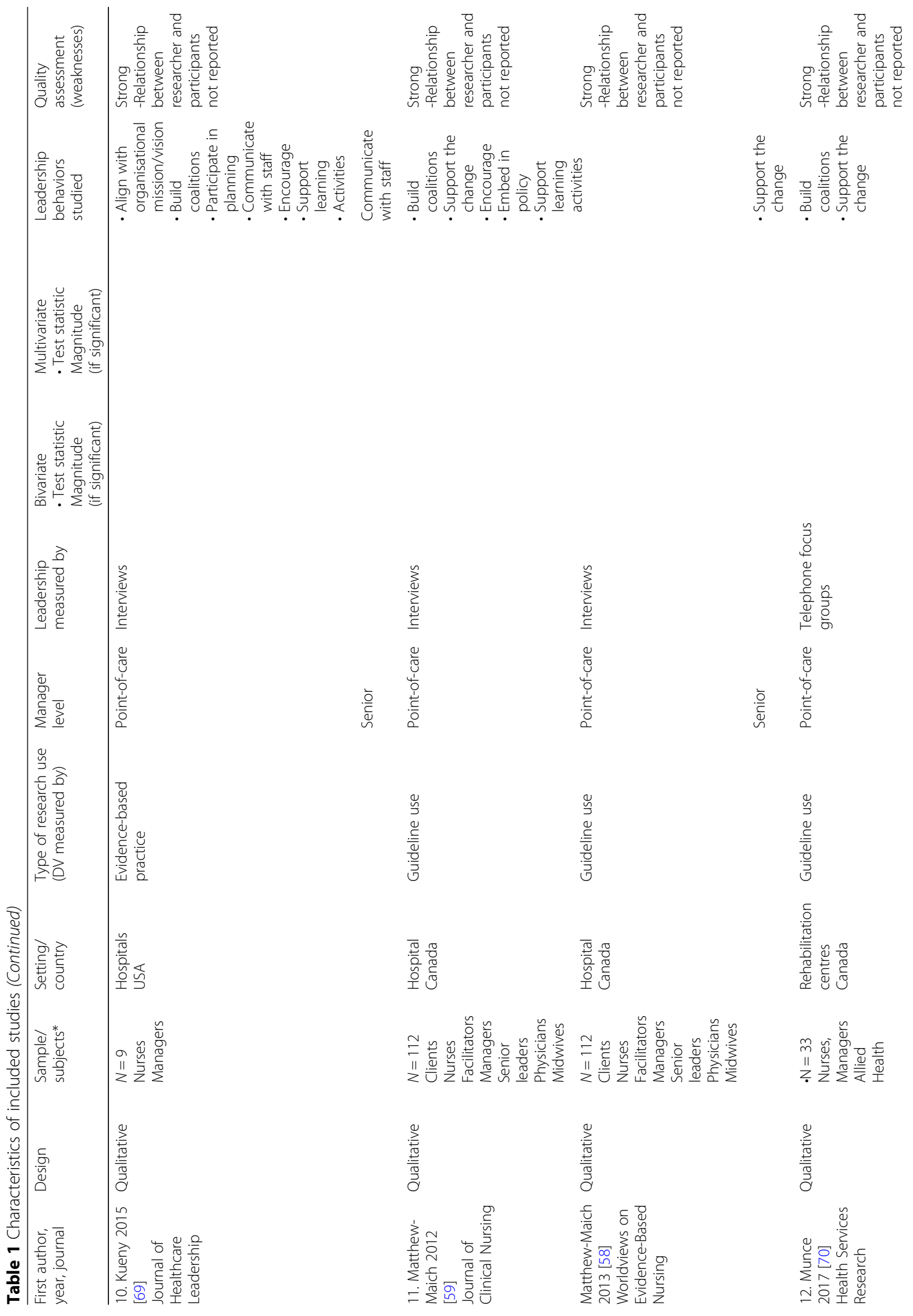




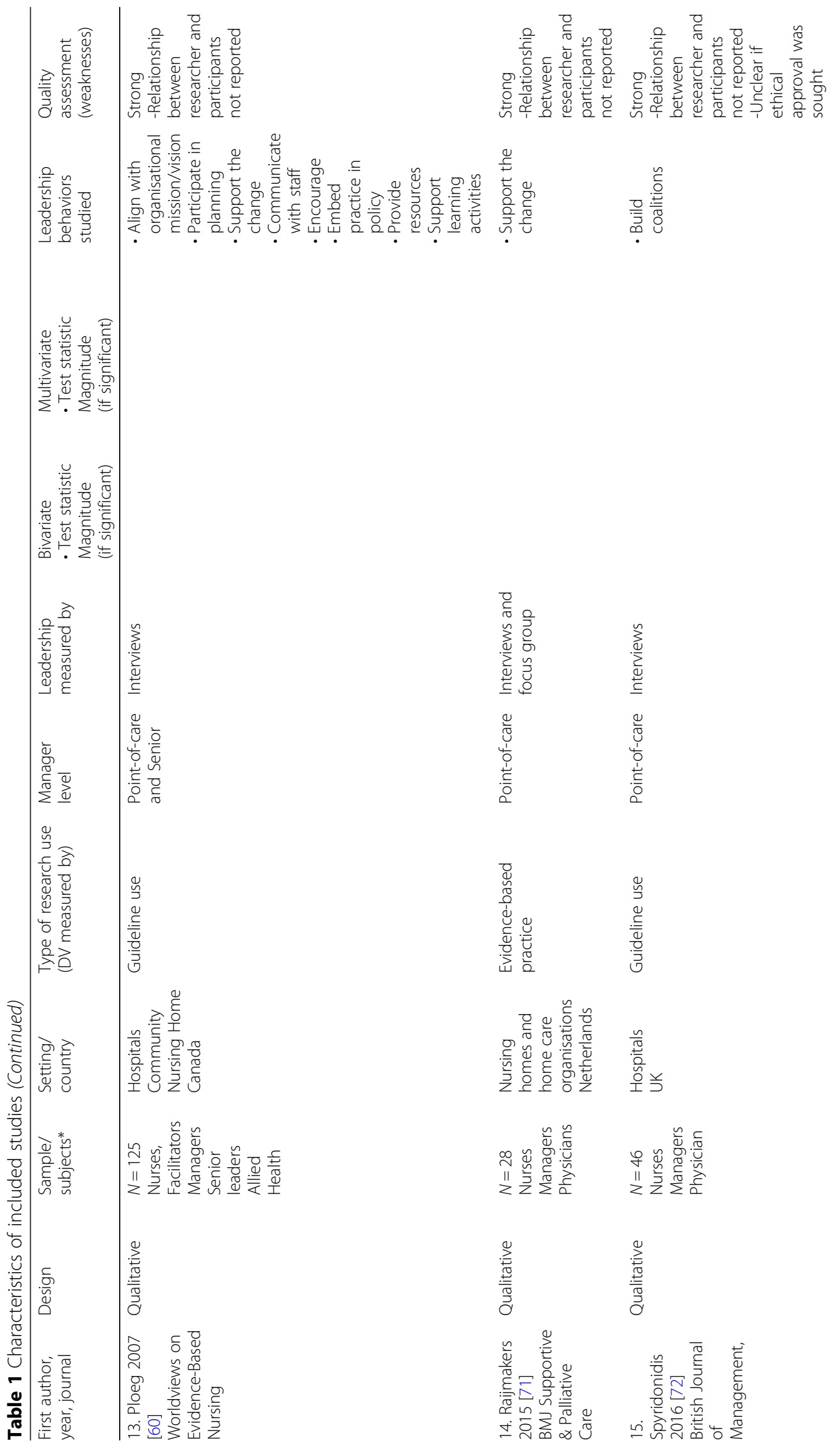




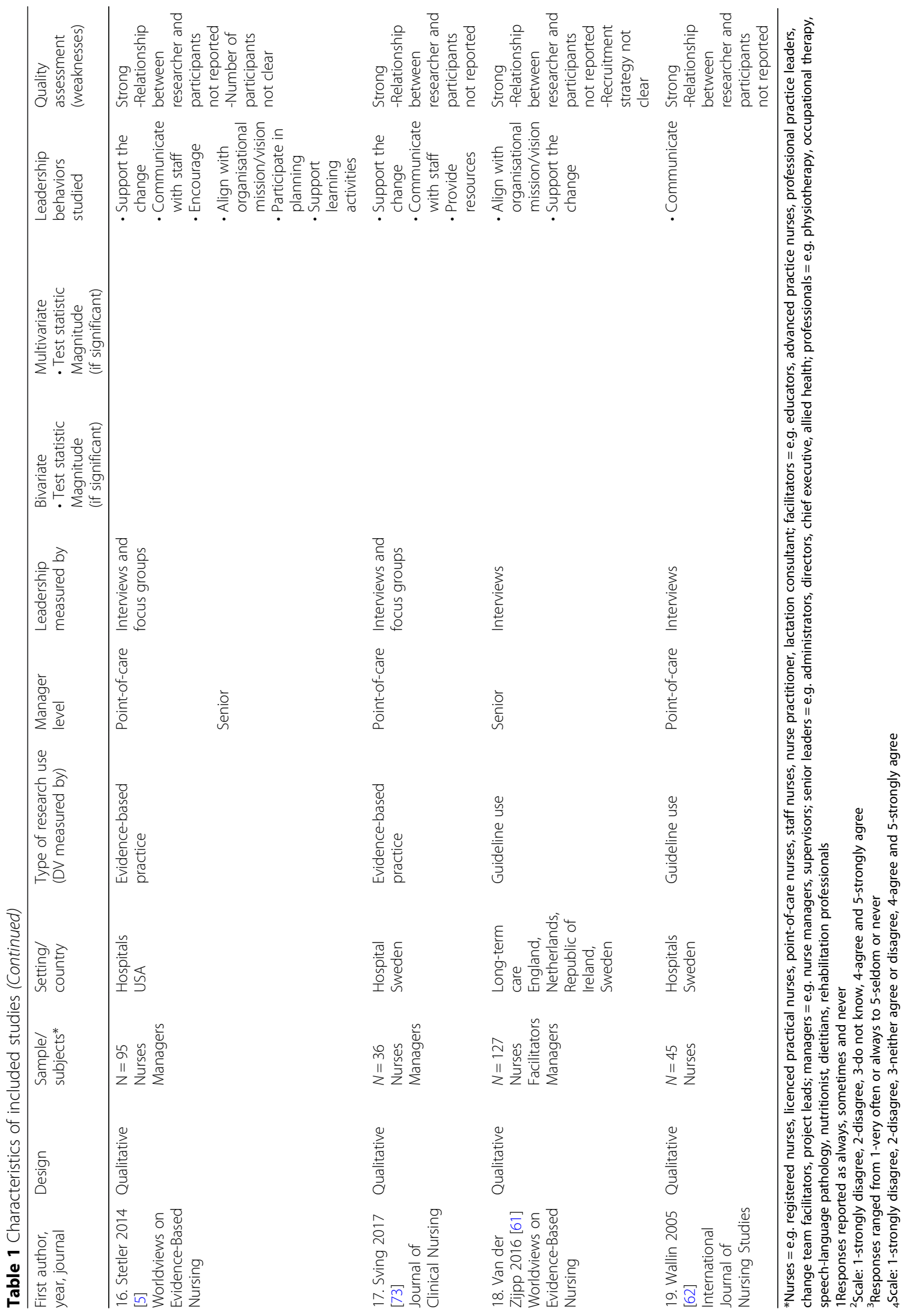


studies, five rated high-moderate [42-45, 50], two low-moderate $[47,49]$, and one weak [51]. Both intervention studies rated high-moderate [31, 52]. Discrepancies in quality assessment mainly related to sample representativeness, response rates, reliability and validity of the dependent variable, and treatment of missing data.

\section{Associations between leadership and research use}

Quantitative studies that evaluated associations between measures of managerial leadership and research use had mixed results. Four leadership measures were statistically significant for point-of-care managers (support [43, 45]; empowering leadership [45]; fair leadership [45]; emotionally intelligent leadership [49], and two measures were not (support [44, 50]; emotionally intelligent leadership [46-48]). For example, Bostrom et al. [43] showed via multivariate analysis $(p=0.044)$ that support from point-of-care managers using the RUQ was significantly related to nurses' use of research findings; however, in another study using the same instrument, managers' support was not significantly related to research use in participants who scored as research users compared to non-research users [44]. Using the QPS Nordic scale, higher leadership scores were significantly correlated to increased research use in multivariate analysis $(p<0.005)$ [45]. For senior managers, three leadership measures were statistically significant (support [42]; provide resources [51]; provide training [51]).

Three of four studies that tested an association between leadership measured with the ACT and research use showed non-significant results when more rigorous tests were performed. Estabrooks et al. [46] demonstrated statistically significant $(p<.05)$ correlations between research use and leadership scores with Pearson's correlation; however, a relationship was not demonstrated with an ANOVA measuring increasing levels of research use and leadership scores. Yamada et al. [49] showed that leadership significantly moderated the effect of research use and pain intensity in in hospitalized children.

Studies including a leadership intervention for unit level managers [31, 52] both demonstrated significant differences in research use scores before and after the intervention, with qualitative data providing insights about leadership behaviours used by managers. In the two quantitative studies measuring senior managers' leadership [42, 51], statistical significance was demonstrated between leadership and research-based practices.

\section{Leadership behaviours}

Twelve leadership behaviours for point-of-care managers were studied in association with research use by clinical staff, and ten for senior managers. Ten of the 12 behaviours for point-of-care managers demonstrated a positive association with research use that were supported by both a qualitative and quantitative or mixed-methods studies. One behaviour that was statistically significant in a quantitative study (distributes work fairly) [45] did not emerge in the qualitative data. All senior managers' behaviours emerged from qualitative data with four of those behaviours (40\%) also supported by quantitative or mixed-methods studies.

For allied health professionals, three behaviours were identified for point-of-care managers and four behaviours for senior managers. Table 2 provides a complete list of the leadership behaviours studied in association with research use by nurses and allied health professionals. Together, behaviours encompassed change-oriented, relations-oriented and task-oriented leadership behaviours.

\section{Change-oriented leadership behaviours}

The most commonly cited behaviour for point-of-care and senior managers was supporting the change that involved being conceptually and operationally committed to research-based practices $[5,31,43,53,55-61,64,70$, 71,73 . Point-of-care managers also ensured that messages about research-based care were consistent with organisational directions and senior leaders' expectations for performance $[54,55,60,63,65,66,69]$, while senior managers engaged in strategic behaviours to reinforce research-based practices as part of the organisation's mission or philosophy $[5,55,60,63,72]$.

Both point-of-care and senior managers built coalitions with inter-professional colleagues, for example, by negotiating with medical staff to change routine orders [53] and working cooperatively with other departments or nurse specialists $[31,55,57-59,63-65,68-70,72]$. Point-of-care managers were involved in planning implementation activities and establishing strategies that aligned to clinical realities so staff could use research evidence in practice $[5,31,54,57,63-66,69]$.

\section{Relations-oriented leadership Behaviours}

Point-of-care managers communicated with staff, giving and seeking information about reasons for change, goals to achieve and audit results [52, 54, 55, 61, 62]. They used targeted language about using research evidence in practice [5], encouraged staff to ask questions and voice concerns [45] while incorporating discussions about research-based practices into group shift reports [54, 58]. They provided clear and explicit reasons research-based practice changes would improve practice, addressing individual concerns and actively encouraging staff while acknowledging efforts to change $[5,31,55,58,61,63,64,69]$.

Relations-oriented leadership behaviours of senior managers emerged in two qualitative studies $[55,60]$. Senior managers communicated and encouraged staff by 
Table $\mathbf{2}$ Leadership behaviours studied in association with research use by clinical staff

\begin{tabular}{|c|c|c|c|c|}
\hline Point-of-care managers' leadership behaviour & $\begin{array}{l}\text { Quantitative studies } \\
(n=8)\end{array}$ & $\begin{array}{l}\text { Mixed methods } \\
(n=2)\end{array}$ & $\begin{array}{l}\text { Qualitative studies } \\
(n=15)\end{array}$ & $\begin{array}{l}\text { Total no. } \\
(n=26)\end{array}$ \\
\hline \multicolumn{5}{|l|}{ Change-oriented leadership behaviours } \\
\hline - Align with organisational mission/vision & - & - & 6 & 6 \\
\hline - Build coalitions with inter-professional colleagues & - & 1 & 8 & 9 \\
\hline - Participate in planning implementation strategies & - & 1 & 6 & 7 \\
\hline - Support the change & $4[2+/ 2-]$ & 1 & 13 & 18 \\
\hline \multicolumn{5}{|l|}{ Relation-oriented leadership behaviours } \\
\hline - Communicate with staff & $1[-]$ & 2 & 8 & 11 \\
\hline • Encourage & $1[+]$ & 1 & 7 & 9 \\
\hline • Emotionally intelligent leadership & $4[1+/ 3-]$ & - & - & 4 \\
\hline \multicolumn{5}{|l|}{ Task-oriented leadership behaviours } \\
\hline - Embed practices in policy & - & - & 3 & 3 \\
\hline - Distribute work fairly & $1[+]$ & - & - & 1 \\
\hline - Monitor indicators & - & 1 & 5 & 6 \\
\hline - Provide resources & - & 1 & 3 & 4 \\
\hline - Support learning activities & - & 1 & 6 & 7 \\
\hline Senior managers' leadership behaviours & $\begin{array}{l}\text { Quantitative studies } \\
(n=1)\end{array}$ & $\begin{array}{l}\text { Mixed methods } \\
(n=1)\end{array}$ & $\begin{array}{l}\text { Qualitative studies } \\
(n=11)\end{array}$ & $\begin{array}{l}\text { Total no. } \\
(n=13)\end{array}$ \\
\hline \multicolumn{5}{|l|}{ Change-oriented leadership behaviours } \\
\hline - Align with organisational mission/vision & - & - & 5 & 5 \\
\hline - Build coalitions with inter-professional colleagues & - & - & 4 & 4 \\
\hline - Participate in planning implementation & - & - & 2 & 2 \\
\hline - Support the change & $1[+]$ & - & 7 & 8 \\
\hline \multicolumn{5}{|l|}{ Relation-oriented leadership behaviours } \\
\hline - Communicate with staff & - & - & 3 & 3 \\
\hline - Encourage & - & - & 2 & 2 \\
\hline \multicolumn{5}{|l|}{ Task-oriented leadership behaviours } \\
\hline - Embed practice in policies & $1[+]$ & - & 3 & 4 \\
\hline - Monitor indicators & - & - & 1 & 1 \\
\hline - Provide resources & - & $1[+]$ & 1 & 2 \\
\hline - Support learning activities & - & $1[+]$ & 3 & 4 \\
\hline
\end{tabular}

$[+]$ association statistically significant, $[-]$ association not statistically significant

articulating support and addressing concerns about research use in practice.

\section{Task-oriented leadership behaviours}

Task-oriented leadership behaviours involved point-of-care and senior managers embedding specific research-based practices into policies [31, 42, 56, 59, 60, 63, 64], providing necessary equipment and supplies [31, 51, 60, 61, 73], supporting learning activities $[51,53-55,59,60]$ and monitoring indicators of research-based practices $[31,54,55,57$, 63-65]. Distributing work fairly, measured on the QPS Nordic Scale, involved distributing work impartially and treating others equally and was higher in units with more research-based care $(p<0.005)$ [45].

\section{Discussion}

\section{Summary of findings}

This systematic review examined qualitative and quantitative evidence on associations between managers' leadership behaviours and nurses and allied health professionals' use of research evidence in clinical practice. Most of the studies were conducted in North America and Europe. No studies focused exclusively on allied health professionals, and only six of the 31 studies reviewed included allied health professionals, offering little empirical evidence for their leadership behaviours that support research use. However, all included studies involved nurses and this body of evidence provides empirical support for a range of leadership behaviors. 
Twelve leadership behaviors had been studied in association with research use, and 11 of these indicated a positive trend towards influencing professional staff to use research evidence in clinical practice.

Since the 2007 review on managerial leadership for nurses' use of research evidence [32]), 19 more studies have been published with a greater number of leadership behaviours identified and a stronger association established with research use. It should be remembered that, although the study purposes were similar, this review had different inclusion criteria. In the current review, a statistical link was required between a leadership variable and research use whereas in the 2007 review, descriptions of variables met inclusion. In addition, implementation of research evidence must have explicitly occurred in the qualitative studies in the current review rather than speculatively explored as in the 2007 review. The current review provides more robust evidence for a greater number of leadership behaviours, increasing understanding of the relationship between leadership and research use. For example, in the past 10 years, evidence has emerged on the importance of managers aligning research use with an organisation's mission, building coalitions with interprofessional colleagues, and being involved in planning implementation strategies. Further evidence has also accumulated on the importance of managers providing support, embedding research evidence in policy and monitoring implementation.

In this synthesis, studies with qualitative $(n=19)$ and mixed-methods $(n=3)$ designs contributed more information about how leaders influenced research use than quantitative studies $(n=9)$. However, data extracted from quantitative studies did not always align with themes extracted from the qualitative data and vice-versa. For example, the measures of emotionally intelligent leadership [41, 53, 54] and fair leadership [45] emerged in quantitative studies only. Different conceptualizations of leadership in research instruments may partially account for the low number of quantitative studies that provided information on leadership behaviours. The QPS Nordic scale, used by Boström et al. [45], measured three aspects of leadership (social support, encouragement, fair leadership) whereas the Alberta Context Tool (ACT), used by Estabrooks et al. [46], Förberg et al. [47] and Squires et al. [48], had a single score representing emotionally intelligent leadership. While two of the individual items in the ACT leadership subscale aligned with our findings (communicates with staff and encourages staff), these items were not individually measured and could not be synthesized separately into our findings. Consistent measurement tools that specifically capture leadership behaviours for research use are necessary to enable meta-analysis in future systematic reviews.

\section{Multidimensional nature of leadership}

Data support the multidimensional nature of leadership and its alignment with behavioural leadership theory [13-15] and concepts of transformational and transactional leadership theory $[16,17]$. Transformational leadership is the degree to which a leader inspires and motivates others to follow an ideal or a particular course of action [16], while transactional leadership focuses on incentives and rewards to meet quality standards [17]. Our findings show that managerial leadership, for both point-of-care and senior managers, inspire, encourage and provide incentives for staff through a combination of change, relations and task-oriented behaviours that are responsive to specific clinical contexts and situations. These behaviours are consistent with transformational and transactional leadership approaches and support the multidimensional nature of implementation leadership previously reported [74-76].

\section{Collaborative activities}

The change and relation-oriented behaviours of building coalitions, participating in planning and communicating with staff reveal an interdependent staff/manager relationship. Managers used integrated strategies within and outside their units to build a sense of community and a culture that supports research use. Findings revealed that point-of-care managers do more than encourage staff to conduct specific tasks and follow policies; they also engaged in tailored exchanges within and across departments and disciplines that influenced the work environment and promoted research use. Managers' priorities and what they pay attention to can signal organisational priorities to staff and directly influence the work environment culture [77].

Our findings highlight managers' use of collaborative approaches such as building coalitions with interprofessional colleagues, to foster staff's use of research in routine practice. This involved negotiating, working cooperatively and engaging actively in collaborative activities. A social network analysis in a Canadian public health department found that managers were central to knowledge flow, interactions and inter-personal connections with staff seeking information about practice [78]. With multidisciplinary collaborations' importance for highquality outcomes in healthcare settings [79], managers play an important role in fostering these collaborations to support staff use research in clinical practice.

\section{A common message}

Our systematic review provides further evidence of manager/staff dyads being influential in translating research evidence into action [69, 80, 81]. Moreover, leadership for research use extends beyond a leader-follower exchange to include change and task-oriented behaviours 
that influence the work environment through organisational structures and processes such as aligning with the organisational mission or vision, embedding in policy, and providing resources. This builds on conceptualization of leadership as meso and macro-level activities that influence individuals, the work environment milieu and organisational infrastructure to move towards goals $[55,82,83]$.

Schein [77] describes a leader's focus and how they communicate priorities as 'primary embedding mechanisms' which are powerful tools to create a work environment for change. Our qualitative findings highlighted that point-of-care and senior managers aligned the concept of research use to a broader organisational mission or vision, signalling to staff the macro-level leadership support for research use in the organisation. Aarons et al. (2016) similarly showed that coordinated and concerted support from leaders at multiple organisational levels, including a common message that links research use to the organisation's mission, vision, values, and operations, contributed to successful implementation and sustained research use in social services organisations [9].

\section{Context of settings}

The small heterogeneous sample in this review did not allow for comparisons across countries, professional groups or clinical settings. Leadership behaviours that most frequently emerged were communication, encouragement, supporting the change and supporting learning activities. The relevance of our findings to other cultural contexts is, however, unclear, particularly where management and leadership conceptualisations may differ with expressions of individuality and social desirability [84]. For example, integrating indigenous ways of knowing is fundamental to using research in healthcare practices in indigenous communities in Canada and involves the participation of community leaders, chiefs and elders [85]. Indigenous people have a long and established history of translating their own knowledge into actions [86] and managers working with indigenous communities must consider nurses and allied health professional's use of research evidence within the broader context of colonisation, discrimination and historical trauma. It is unclear how leadership behaviours from this review translate to different global or cultural contexts.

\section{Inter-professional implications}

An increase in published reports over the past 5 years suggests that managerial leadership is gaining attention as an area of study. Note, however, that all studies involved nurses and only six included allied health professionals. While 'allied health professionals' can include different professional groups, dependent on where and who is defining them [29], we chose to only focus on physiotherapy, occupational therapy, speech-language pathology, and dietitians as they are central to the delivery of health care services alongside nursing and medicine. While a positive association has been established between leadership and social services workers' research use in community mental health settings and child welfare social services [87] [9], these studies did not meet inclusion criteria in this review. However, Aarons et al. [9] and Aarons and Sawitzky [87] findings are consistent with ours, demonstrating the full range of leadership behaviours that influence the acceptance and use of research evidence in clinical practice.

With few studies including allied health professionals, little can be extrapolated from the data regarding their managers' leadership. Although allied health professionals are part of an interdisciplinary team with a professional obligation to incorporate the best available research evidence into their practices, their organisation of care is typically more independent than nurses. Our findings may have limited transferability to leadership directions of managers working with allied health professionals.

\section{Methodological implications for future research}

To increase confidence in future study results examining managerial leadership and research use, methodologies with higher internal and external validity are required. To move the science forward and develop interventions that improve the quality of patient care, five future research implications are noted.

First, research is needed to understand the conceptual similarities and differences between leadership behaviors identified in this review, including studies exploring leadership in different cultural contexts to expand implementation leadership theory. Second, building on conceptual development of leadership for research use, there is a need for consistent measures across studies as only two instruments were used in multiple studies in our review (Research Utilization Questionnaire [43, 44] and Alberta Context Tool [46-48]) and the absence of common measures makes it difficult to build a strong body of knowledge. Using consistent measures will allow findings to be pooled for meta-analysis and sub-group analysis to determine the leadership practices required to facilitate staff use research evidence in different professional groups and sectors.

Third, while our findings are important to understanding how managers and staff perceive leadership for research use, robust methodological studies are now required to determine behaviours that predict nurses' and allied health professionals' research use and develop theory-based leadership interventions to improve the quality of patient care. Fourth, since only six studies were found that included allied health professionals and no studies focusing exclusively on them, there is a pressing need for research on managerial leadership with 
allied health professionals. Finally, studies are needed to understand the conditions that support managers to effectively facilitate and support staff, including conditions that help managers integrate and use research evidence in their management decision-making.

\section{Limitations}

Despite employing rigorous methods in conducting this review, it has limitations that must be acknowledged. While reference lists of included studies were examined for other literature, we did not search gray literature databases, so our search was restricted to primary research in peer-reviewed journals and might have missed relevant unpublished research. Moreover, we did not contact primary authors of excluded studies and may have excluded articles with unclear details of managerial roles. Studies published in languages other than those of the research team (English and French) were also excluded, and databases that could not be accessed in English, such as the Chinese databases CNKI (中国知网) or WANFANG (万方数据库), were not searched. Finally, methodological strengths and weaknesses were not considered while determining our conclusions. Instead, all studies were synthesized equally while reporting on the methodological quality to provide a literature summary and show the current evidence baseline clearly.

\section{Conclusion}

This systematic literature review suggests that managers use a range of leadership practices involving change, relations and task-oriented behaviours to facilitate and support nursing and allied health staff use research evidence use in their clinical practice. While empirical research on allied health professionals is limited, all studies included nurses with a consistent trend across studies that highlighted managers' commitment, engagement, communication and support. Changeoriented behaviours involve gaining commitment to a broader vision and building coalitions to support the vision, relation-oriented behaviours encompass interpersonal relationships to encourage and support staff, and task-oriented behaviours include concrete activities to operationalize the vision like supporting learning, monitoring performance and outcomes and ensuring policies reflect research-based practices. More robust research designs that include consistent and valid leadership measures specifically for research use are required to advance implementation science on leadership.

\section{Additional file}

Additional file 1: Managerial leadership for research use in nursing and allied health care: search strategies. (PDF $32 \mathrm{~kb}$ )

\section{Abbreviations}

ACT: Alberta Context Tool; CASP: Critical Appraisal Skills Programme; LPN: Licensed practical nurse; OTs: Occupational therapists; PTs: Physiotherapists; RN: Registered nurse; RPN: Registered practical nurses; RUQ: Research Utilization Questionnaire; SLPS: Speech-language pathologists

\section{Acknowledgements}

We would like to thank Canadian Institutes for Health Research (CIHR) (Knowledge Synthesis Grant FRN 132036) for providing the funding for this project. We would also like to thank the graduate students, trainees and research staff at the University of Ottawa and Saint Elizabeth who helped with screening, data extraction, content analysis coding and quality appraisal: Sayna Bahraini, Seham Rabaa, Ovini Thomas, and Mirella Maria Soares Veras. At the time of this work, Dr. Squires holds a CIHR New Investigator Award and a University Research Chair in Health Evidence Implementation and Dr. Brosseau holds a University Research Chair in Evidence-based Practice in Rehabilitation. The authors accept full responsibility for the manuscript. Funders were not involved in the conduct of the study or preparation of the manuscript.

\section{Funding}

This systematic review is a knowledge synthesis project funded by the Canadian Institute of Health Research Funding Reference Number (FRN) 132036.

Availability of data and materials

The datasets used and/or analysed during the current study are available from the corresponding author on reasonable request.

\section{Authors' contributions}

WG conceptualized and secured funding for this study from the Canadian Institutes for Health Research (CIHR). She designed and led the study and drafted the manuscript. LB, ME, IDG, JES and LW assisted with study design, analysis of data and interpretation of data. In addition, IDG and JES provided methodological consultation. MCD conducted the search strategy. All authors contributed to interpretation of findings and edited, read and approved the final manuscript. PH, LAA and JMC were knowledge users for this study.

Ethics approval and consent to participate

As this paper is a synthesis of published literature, ethics approval was not required.

\section{Consent for publication}

Not applicable

\section{Competing interests}

Gifford, Graham, Squires and Wallin were the authors on studies included in this review. The other authors declare that they have no competing interests.

\section{Publisher's Note}

Springer Nature remains neutral with regard to jurisdictional claims in published maps and institutional affiliations.

\section{Author details}

${ }^{1}$ Faculty of Health Sciences, School of Nursing, University of Ottawa, Ottawa, Ontario, Canada. ${ }^{2}$ Clinical Epidemiology Program, The Ottawa Hospital Research Institute, Ottawa, Ontario, Canada. ${ }^{3}$ Telfer School of Management, University of Ottawa, Ottawa, Ontario, Canada. ${ }^{4}$ Canadian Nurses Association, Ottawa, Ontario, Canada. ${ }^{5}$ Faculty of Health Sciences, School of Rehabilitation Sciences, University of Ottawa, Ottawa, Ontario, Canada. ${ }^{6}$ Canadian Association of Occupational Therapists, Ottawa, Ontario, Canada. ${ }^{7}$ Health Sciences Library, University of Ottawa, Ottawa, Ontario, Canada. ${ }^{8}$ Elisabeth Bruyere Research Institute, Ottawa, Ontario, Canada. ${ }^{9}$ SE Research Centre, SE Health, Markham, Ontario, Canada. ${ }^{10}$ Faculty of Health, School of Nursing, York University, Toronto, Ontario, Canada. ${ }^{11}$ School of Education, Health and Social Studies, Dalarna University, Falun, Sweden. ${ }^{12}$ Sahlgrenska Academy, Department of Health Care Sciences, University of Gothenburg, Gothenburg, Sweden. ${ }^{13}$ Department of Neurobiology, Care Sciences and Society, Division of Nursing Karolinska Institutet, Stockholm, Sweden. ${ }^{14}$ School of Epidemiology and Public Health, University of Ottawa, Ottawa, Ontario, Canada. 
Received: 1 February 2018 Accepted: 10 September 2018 Published online: 27 September 2018

\section{References}

1. Lobb R, Colditz GA. Implementation science and its application to population health. Annu Rev Public Health. 2013;34:235-51. https://doi.org/10.1146/ annurev-publhealth-031912-114444.

2. Melnyk BM, Fineout-Overholt E. Evidence-based practice in nursing \& healthcare : a guide to best practice. 2nd ed. Philadelphia: Wolters Kluwer/ Lippincott Williams \& Wilkins Health; 2011.

3. Erasmus V, Daha TJ, Brug H, Richardus JH, Behrendt MD, Vos MC, et al. Systematic review of studies on compliance with hand hygiene guidelines in hospital care. Infect Control Hosp Epidemiol. 2010;31(3):283-94. https:// doi.org/10.1086/650451.

4. Rello J, Lorente C, Bodi M, Diaz E, Ricart M, Kollef MH. Why do physicians not follow evidence-based guidelines for preventing ventilator-associated pneumonia?: a survey based on the opinions of an international panel of intensivists. Chest. 2002;122(2):656-61.

5. Stetler CB, Ritchie JA, Rycroft-Malone J, Charns MP. Leadership for evidence-based practice: strategic and functional behaviors for institutionalizing EBP. Worldviews Evid-Based Nurs. 2014;11(4):219-26. https://doi.org/10.1111/wvn.12044.

6. Burnes B. Emergent change and planned change - competitors or allies? the case of XYZ construction. Int J Oper Prod Manag. 2004;24(9):886-902.

7. Jennings C, Fitzsimons D, Kerins M, McKee G. What's preventing nurses and allied professionals from implementing clinical guidelines? Results of a survey from the European Society of Cardiology Council on Cardiovascular Nursing and Allied Professions. Eur J Cardiovasc Nursing. 2016;15:195. https://doi.org/10.1177/1474515116635947.

8. Rycroft-Malone J, Seers K, Chandler J, Hawkes CA, Crichton N, Allen C, et al. The role of evidence, context, and facilitation in an implementation trial: implications for the development of the PARIHS framework. Implement Sci. 2013;8:28. https://doi.org/10.1186/1748-5908-8-28.

9. Aarons GA, Green AE, Trott E, Willging CE, Torres EM, Ehrhart MG, et al. The roles of system and organizational leadership in system-wide evidencebased intervention sustainment: a mixed-method study. Admin Pol Ment Health. 2016;43(6):991-1008. https://doi.org/10.1007/s10488-016-0751-4.

10. Sackett D, Richardson W, Rosenberg W, Haynes R. Evidence-based medicine: how to practice and teach EBM. Churchill Livingstone, New York, NY. Edinburgh, UK. 1996.

11. Harvey G, Fitzgerald L, Fielden S, McBride A, Waterman H, Bamford D, et al. The NIHR Collaborations for Leadership in Applied Health Research and Care (CLAHRC) for Greater Manchester: combining empirical, theoretical and experiential evidence to design and evaluate a large-scale implementation strategy. Implement Sci. 2011;6:96. https://doi.org/10.1186/1748-5908-6-96.

12. House R, Hanges P, Javidan M, Dorfam P, Gupta V. Culture, leadership, and organizations: the GLOBE study of 62 societies. Thousand Oaks: Sage; 2004

13. Yukl G. Leadership in organizations. 8th ed. Upper Saddle River: Pearson; 2013.

14. Yukl G, Gordon A, Taber T. A hierarchical taxonomy of leadership behavior: integrating a half century of behavior research. J Leadersh Org Stud. 2002; 19:15-32.

15. Ekvall G, Arvonen J. Change-centered leadership: an extension of the twodimensional model. Scand J Manag. 1991;7(1):17-26.

16. Bass B. Two decades of research and development in transformational leadership. Eur J Work Organ Psychol. 1999;8:9-32.

17. Avolio B, Bass B, Jung D. Re-examining the components of transformational and transactional leadership using the Multifactor Leadership Questionnaire. J Occup Organ Psychol. 1999;72(4):441-62

18. Khammarnia M, Haj Mohammadi M, Amani Z, Rezaeian S, Setoodehzadeh F. Barriers to implementation of evidence based practice in Zahedan teaching hospitals, Iran, 2014. Nurs Res Pract. 2015;2015:357140. https://doi.org/10. $1155 / 2015 / 357140$

19. Majid S, Foo S, Luyt B, Zhang X, Theng YL, Chang YK, et al. Adopting evidence-based practice in clinical decision making: nurses' perceptions, knowledge, and barriers. J Med Libr Assoc. 2011;99(3):229-36. https://doi org/10.3163/1536-5050.99.3.010.

20. Stavor DC, Zedreck-Gonzalez J, Hoffmann RL. Improving the use of evidence-based practice and research utilization through the identification of barriers to implementation in a critical access hospital. J Nurs Adm. 2017; 47(1):56-61. https://doi.org/10.1097/NNA.0000000000000437.

21. Ubbink DT, Guyatt GH, Vermeulen H. Framework of policy recommendations for implementation of evidence-based practice: a systematic scoping review. BMJ Open. 2013;3(1). https://doi.org/10.1136/ bmjopen-2012-001881.

22. Mintzberg $H$. The nature of managerial work. New York: Harper \& Row; 1973.

23. McCormack B, Rycroft-Malone J, Decorby K, Hutchinson AM, Bucknall T, Kent $B$, et al. A realist review of interventions and strategies to promote evidence-informed healthcare: a focus on change agency. Implement Sci. 2013;8:107. https://doi.org/10.1186/1748-5908-8-107.

24. Stetler CB, Ritchie JA, Rycroft-Malone J, Schultz AA, Charns MP. Institutionalizing evidence-based practice: an organizational case study using a model of strategic change. Implement Sci. 2009;4:78. https://doi. org/10.1186/1748-5908-4-78.

25. Jeans M, Rowat K. Competencies required of nurse managers. Ottawa: Canadian Nurses Association; 2004

26. World Health Organization. Health topics: nursing. 2017. http://www.who. int/topics/nursing/en/. Accessed 19 Oct 2017.

27. Canadian Nurses Association. Framework for the practice of registered nurses in Canada. Ottawa: Canadian Nurses Association; 2015.

28. Grimmer-Somers K, Kumar S, Worley A, Young A. Practical tips in finding the evidence: an allied health primer. Manila: UST publishing house; 2009.

29. HLWIKI_Canada_Contributors. Allied health professionals. HLWIKI Canada. 2016. http://hlwiki.slais.ubc.ca/index.php?title=Allied_health_ professionals\&oldid=144214. Accessed 21 Aug 2018.

30. Gifford WA, Holyoke P, Squires JE, Angus D, Brosseau L, Egan M, et al. Managerial leadership for research use in nursing and allied health care professions: a narrative synthesis protocol. Syst Rev. 2014;3:57. https://doi. org/10.1186/2046-4053-3-57

31. Gifford WA, Davies BL, Graham ID, Tourangeau A, Woodend AK, Lefebre N. Developing leadership capacity for guideline use: a pilot cluster randomized control trial. Worldviews Evid-Based Nurs. 2012;10(1):51-65. https://doi.org/ 10.1111/j.1741-6787.2012.00254.x

32. Gifford W, Davies B, Edwards N, Griffin P, Lybanon V. Managerial leadership for nurses' use of research evidence: an integrative review of the literature. Worldviews Evid-Based Nurs. 2007:4(3):126-45. https://doi.org/10.1111/j. 1741-6787.2007.00095.x.

33. Grimshaw J. A guide to knowledge synthesis: a knowledge synthesis chapter. Knowledge Translation: Canadian Institutes of Health Research; 2010.

34. Amara N, Ouimet M, Landry R. New evidence on instrumental, conceptual, and symbolic utilization of university research in government agencies. Sci Commun. 2004;26:75-106.

35. Estabrooks C, Wallin L, Milner M. Measuring knowledge utilization in health care. Int J Policy Analysis Eval. 2003;1:3-36.

36. Dawes M, Summerskill W, Glasziou P, Cartabellotta A, Martin J, Hopayian K, et al. Sicily statement on evidence-based practice. BMC Med Educ. 2005;5(1): 1. https://doi.org/10.1186/1472-6920-5-1.

37. Squires JE, Estabrooks CA, Gustavsson P, Wallin L. Individual determinants of research utilization by nurses: a systematic review update. Implement Sci. 2011;6(1). https://doi.org/10.1186/1748-5908-6-1.

38. Critical Appraisal Skills Programme. 10 questions to help you make sense of qualitative research 2017.

39. Kmet L, Lee R, Cook L. Standard quality assessment criteria for evaluating primary research papers from a variety of fields. Edmonton: Alberta Heritage Foundation for Medical Research; 2004.

40. Estabrooks CA, Cummings GG, Olivo SA, Squires JE, Giblin C, Simpson N. Effects of shift length on quality of patient care and health provider outcomes: systematic review. Qual Saf Health Care. 2009;18(3):181-8. https://doi.org/10.1136/qshc.2007.024232.

41. Popay J, Roberts H, Sowden A, Petticrew M, Arai L, Rodgers M et al. Guidance on the conduct of narrative synthesis in systematic reviews: a product from the ESRC Methods Programme. 2006.

42. Ball K. Surgical smoke evacuation guidelines: compliance among perioperative nurses. AORN J. 2010;92(2):e1-23. https://doi.org/10.1016/j.aorn.2009.10.026

43. Bostrom AM, Wallin L, Nordstrom G. Evidence-based practice and determinants of research use in elderly care in Sweden. J Eval Clin Pract. 2007;13(4):665-73. https://doi.org/10.1111/j.1365-2753.2007.00807.x.

44. Bostrom AM, Kajermo KN, Nordstrom G, Wallin L. Registered nurses' use of research findings in the care of older people. J Clin Nurs. 2009;18(10):143041. https://doi.org/10.1111/j.1365-2702.2008.02370.x.

45. Bostrom AM, Rudman A, Ehrenberg A, Gustavsson JP, Wallin L. Factors associated with evidence-based practice among registered nurses in Sweden: a national cross-sectional study. BMC Health Serv Res. 2013:13:165. https://doi.org/10.1186/1472-6963-13-165. 
46. Estabrooks CA, Squires JE, Cummings GG, Birdsell JM, Norton PG. Development and assessment of the Alberta Context Tool. BMC Health Serv Res. 2009;9:234. https://doi.org/10.1186/1472-6963-9-234.

47. Forberg $U$, Wallin $L$, Johansson $E$, Ygge BM, Backheden M, Ehrenberg A. Relationship between work context and adherence to a clinical practice guideline for peripheral venous catheters among registered nurses in pediatric care. Worldviews Evid-Based Nurs. 2014;11(4):227-39. https://doi. org/10.1111/wvn.12046.

48. Squires JE, Estabrooks CA, Scott SD, Cummings GG, Hayduk L, Kang SH, et al. The influence of organizational context on the use of research by nurses in Canadian pediatric hospitals. BMC Health Serv Res. 2013;13:351. https:// doi.org/10.1186/1472-6963-13-351.

49. Yamada J, Squires JE, Estabrooks CA, Victor C, Stevens B. Pain CTiCs. The role of organizational context in moderating the effect of research use on pain outcomes in hospitalized children: a cross sectional study. BMC health services research. 2017;17(1):68 https://doi.org/10.1186/s12913-017-2029-2.

50. Cummings GG, Hewko SJ, Wang M, Wong CA, Laschinger HKS, Estabrooks CA. Impact of managers' coaching conversations on staff knowledge use and performance in long-term care settings. Worldviews Evid-Based Nurs. 2018;15(1):62-71. https://doi.org/10.1111/wvn.12233.

51. Balbale SN, Hill JN, Guihan M, Hogan TP, Cameron KA, Goldstein B, et al. Evaluating implementation of methicillin-resistant Staphylococcus aureus (MRSA) prevention guidelines in spinal cord injury centers using the PARIHS framework: a mixed methods study. Implement Sci. 2015;10:130. https://doi. org/10.1186/s13012-015-0318-x.

52. Rangachari $P$, Madaio M, Rethemeyer RK, Wagner $P$, Hall L, Roy S, et al. The evolution of knowledge exchanges enabling successful practice change in two intensive care units. Health Care Manag Rev. 2015;40(1):65-78. https:// doi.org/10.1097/HMR.0000000000000001.

53. Angus J, Hodnett E, O'Brien-Pallas L. Implementing evidence-based nursing practice: a tale of two intrapartum nursing units. Nurs Inq. 2003;10(4):218-28.

54. Fleiszer AR, Semenic SE, Ritchie JA, Richer MC, Denis JL. A unit-level perspective on the long-term sustainability of a nursing best practice guidelines program: an embedded multiple case study. Int J Nurs Stud. 2016;53:204-18. https://doi.org/10.1016/j.jpurstu.2015.09.004.

55. Gifford WA, Davies B, Edwards N, Graham ID. Leadership strategies to influence the use of clinical practice guidelines. Nurs Leadersh (Tor Ont). 2006;19(4):72-88.

56. Graham ID, Logan J, Davies B, Nimrod C. Changing the use of electronic fetal monitoring and labor support: a case study of barriers and facilitators. Birth. 2004;31(4):293-301. https://doi.org/10.1111/j.0730-7659.2004.00322.x.

57. Ireland S, Kirkpatrick H, Boblin S, Robertson K. The real world journey of implementing fall prevention best practices in three acute care hospitals: a case study. Worldviews Evid-Based Nurs. 2013;10(2):95-103. https://doi.org/ 10.1111/j.1741-6787.2012.00258.x.

58. Matthew-Maich N, Ploeg J, Dobbins M, Jack S. Supporting the uptake of nursing guidelines: what you really need to know to move nursing guidelines into practice. Worldviews Evid-Based Nurs. 2013;10(2):104-15. https://doi.org/10.1111/j.1741-6787.2012.00259.x.

59. Matthew-Maich N, Ploeg J, Jack S, Dobbins M. Leading on the frontlines with passion and persistence: a necessary condition for breastfeeding best practice guideline uptake. J Clin Nurs. 2013;22(11-12):1759-70. https://doi. org/10.1111/jocn.12027.

60. Ploeg J, Davies B, Edwards N, Gifford W, Miller PE. Factors influencing bestpractice guideline implementation: lessons learned from administrators, nursing staff, and project leaders. Worldviews Evid-Based Nurs. 2007;4(4): 210-9. https://doi.org/10.1111/j.1741-6787.2007.00106.x.

61. van der Zijpp TJ, Niessen T, Eldh AC, Hawkes C, McMullan C, Mockford C, et al. A bridge over turbulent waters: illustrating the interaction between managerial leaders and facilitators when implementing research evidence. Worldviews Evid-Based Nurs. 2016;13(1):25-31. https://doi.org/10.1111/ wvn.12138.

62. Wallin L, Rudberg A, Gunningberg L. Staff experiences in implementing guidelines for Kangaroo Mother Care--a qualitative study. Int J Nurs Stud. 2005;42(1):61-73. https://doi.org/10.1016/j.ijnurstu.2004.05.016.

63. Cheng L, Broome ME, Feng S, Hu Y. Factors influencing the implementation of evidence in Chinese nursing practice. J Clin Nurs. 2017;26(23-24):510312. https://doi.org/10.1111/jocn.14053.

64. Cheng L, Feng S, Hu Y, Broome ME. Leadership practices of nurse managers for implementing evidence-based nursing in China. J Nurs Manag. 2018. https://doi.org/10.1111/jonm.12594.
65. Chimeddamba O, Peeters A, Ayton D, Tumenjargal E, Sodov S, Joyce C. Implementation of clinical guidelines on diabetes and hypertension in urban Mongolia: a qualitative study of primary care providers' perspectives and experiences. Implement Sci. 2015;10:112. https:/doi.org/10.1186/s13012-015-0307-0.

66. Fleiszer AR, Semenic SE, Ritchie JA, Richer MC, Denis JL. Nursing unit leaders' influence on the long-term sustainability of evidence-based practice improvements. J Nurs Manag. 2016;24(3):309-18. https://doi.org/10.1111/jonm.12320.

67. Herbert G, Sutton E, Burden S, Lewis S, Thomas S, Ness A, et al. Healthcare professionals' views of the enhanced recovery after surgery programme: a qualitative investigation. BMC Health Serv Res. 2017;17(1):617. https://doi. org/10.1186/s12913-017-2547-y.

68. Higuchi KS, Davies B, Ploeg J. Sustaining guideline implementation: a multisite perspective on activities, challenges and supports. J Clin Nurs. 2017;26(23-24):4413-24. doi:https://doi.org/10.1111/jocn.13770.

69. Kueny A, Shever LL, Lehan Mackin M, Titler MG. Facilitating the implementation of evidence - based practice through contextual support and nursing leadership. J Healthc Leadersh. 2015;7:29-39. https://doi.org/10. 2147/JHL.S45077.

70. SEP M, Graham ID, Salbach NM, Jaglal SB, Richards CL, Eng JJ, et al. Perspectives of health care professionals on the facilitators and barriers to the implementation of a stroke rehabilitation guidelines cluster randomized controlled trial. BMC Health Serv Res. 2017;17(1):440. https://doi.org/10.1186/ s12913-017-2389-7.

71. Raijmakers N, Dekkers A, Galesloot C, van Zuylen L, van der Heide A. Barriers and facilitators to implementation of the Liverpool Care Pathway in the Netherlands: a qualitative study. BMJ Support Palliat Care. 2015;5(3):259-65. https://doi.org/10.1136/bmjspcare-2014-000684.

72. Spyridonidis D, Currie G. The translational role of hybrid nurse middle managers in implementing clinical guidelines: effect of, and upon, professional and managerial hierarchies. Br J Manag. 2016;27(4):760-77.

73. Sving E, Fredriksson L, Gunningberg L, Mamhidir A-G. Getting evidencebased pressure ulcer prevention into practice: a process evaluation of a multifaceted intervention in a hospital setting. J Clin Nurs. 2017;26(19-20): 3200-11. https://doi.org/10.1111/jocn.13668.

74. Aarons GA. Transformational and transactional leadership: association with attitudes toward evidence-based practice. Psychiatr Serv. 2006;57(8):1162.

75. Aarons GA, Ehrhart MG, Farahnak LR. The implementation leadership scale (ILS): development of a brief measure of unit level implementation leadership. Implement Sci. 2014;9(45):10.

76. Gifford WA. Ottawa model of implementation leadership and implementation leadership scale: mapping concepts for developing and evaluating theorybased leadership interventions. J Healthcare Leadership. 2017;9:9.

77. Schein EH. Organizational culture and leadership third edition. Third edition ed. San Francisco: John Wiley \& Sons, Inc; 2004.

78. Yousefi-Nooraie R, Dobbins M, Brouwers M, Wakefield P. Information seeking for making evidence-informed decisions: a social network analysis on the staff of a public health department in Canada. BMC Health Serv Res. 2012;12:118. https://doi.org/10.1186/1472-6963-12-118.

79. Legare F, Stacey D, Graham ID, Elwyn G, Pluye P, Gagnon MP, et al. Advancing theories, models and measurement for an interprofessional approach to shared decision making in primary care: a study protocol. BMC Health Serv Res. 2008;8:2. https://doi.org/10.1186/1472-6963-8-2.

80. Cummings GG, Estabrooks CA, Midodzi WK, Wallin L, Hayduk L. Influence of organizational characteristics and context on research utilization. Nurs Res. 2007;56(4 Suppl):S24-39. https://doi.org/10.1097/01.NNR.0000280629.63654.95.

81. Davies B, Edwards N, Ploeg J, Virani T. Insights about the process and impact of implementing nursing guidelines on delivery of care in hospitals and community settings. BMC Health Serv Res. 2008;8:29. https://doi.org/10. 1186/1472-6963-8-29.

82. Barrett L, Plotnikoff RC, Raine K, Anderson D. Development of measures of organizational leadership for health promotion. Health Educ Behav. 2005; 32(2):195-207. https://doi.org/10.1177/1090198104271970.

83. Barrett LL, Plotnikoff RC, Raine K. Organizational leadership and its relationship to regional health authority actions to promote health. J Health Organ Manag. 2007;21(3):259-82. https://doi.org/10.1108/ 14777260710751735

84. House R, Dorfman P, Javidan M, Hanges P, de Luque M. Strategic leadership behaviour and effectiveness in 24 countries. Thousand Oaks: Sage; 2014.

85. Smylie J, Martin C, Kaplan-Myrth N, Steele L, Tait C, Hogg W. Knowledge translation and indigenous knowledge. Int J Circumpolar Health. 2004: 63(sup2):139-43. 
86. Morton Ninomiya ME, Pollock NJ. Reconciling community-based indigenous research and academic practices: knowing principles is not always enough. Soc Sci Med. 2017;172:28-36. https://doi.org/10.1016/j.socscimed.2016.11.007.

87. Aarons GA, Sawitzky AC. Organizational culture and climate and mental health provider attitudes toward evidence-based practice. Psychol Serv. 2006;3(1):61-72. https://doi.org/10.1037/1541-1559.3.1.61.

Ready to submit your research? Choose BMC and benefit from:

- fast, convenient online submission

- thorough peer review by experienced researchers in your field

- rapid publication on acceptance

- support for research data, including large and complex data types

- gold Open Access which fosters wider collaboration and increased citations

- maximum visibility for your research: over $100 \mathrm{M}$ website views per year

At $\mathrm{BMC}$, research is always in progress.

Learn more biomedcentral.com/submissions 